\title{
Nanoscale
}

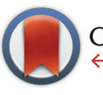

CrossMark \& click for updates

Cite this: Nanoscale, 2016, 8, 2892

Received 17th November 2015,

Accepted 9th January 2016

DOI: $10.1039 / c 5 n r 08122 j$

www.rsc.org/nanoscale

\section{Tuning $\mathrm{Ag}_{29}$ nanocluster light emission from red to blue with one and two-photon excitation $\uparrow$}

\author{
Isabelle Russier-Antoine, ${ }^{a}$ Franck Bertorelle, ${ }^{a}$ Ramzi Hamouda, ${ }^{a}$ Driss Rayane, ${ }^{a}$ \\ Philippe Dugourd, ${ }^{a}$ Željka Sanader, ${ }^{b, c}$ Vlasta Bonačić-Koutecký, ${ }^{b, d}$ \\ Pierre-François Brevet ${ }^{a}$ and Rodolphe Antoine*a
}

\begin{abstract}
We demonstrate that the tuning of the light emission from red to blue in dihydrolipoic acid (DHLA) capped $\mathrm{Ag}_{29}$ nanoclusters can be trigged with one and two photon excitations. The cluster stoichiometry was determined with mass spectrometry and found to be $\mathrm{Ag}_{29}(\mathrm{DHLA})_{12}$. In a detailed optical investigation, we show that these silver nanoclusters exhibit a strong red photoluminescence visible to the naked eye and characterized by a quantum yield of nearly 2\% upon one-photon excitation. In the nonlinear optical (NLO) study of the properties of the clusters, the two-photon excited fluorescence spectra were recorded and their first hyperpolarizability obtained. The two-photon absorption cross-section at $\sim 800 \mathrm{~nm}$ for $\operatorname{Ag}_{29}(\mathrm{DHLA})_{12}$ is higher than $10^{4} \mathrm{GM}$ and the hyperpolarizability is $106 \times 10^{-30}$ esu at the same excitation wavelength. The two-photon excited fluorescence spectrum appears strongly blue-shifted as compared to the one-photon excited spectrum, displaying a broad band between 400 and $700 \mathrm{~nm}$. The density functional theory (DFT) provides insight into the structural and electronic properties of $\mathrm{Ag}_{29}(\mathrm{DHLA})_{12}$ as well as into interplay between metallic subunit or core and ligands which is responsible for unique optical properties.
\end{abstract}

\section{Introduction}

Atomically precise silver nanoclusters (Ag NCs) have emerged as fascinating fluorescent nanomaterials and attracted considerable research interest in both fundamental research and practical applications, including biosensing, ${ }^{1}$ bioimaging, ${ }^{2}$ catalysis, ${ }^{3}$ or photonics. ${ }^{4}$ Due to enhanced quantum confinement, in particular, they exhibit new optical properties which are observed neither in molecules nor in bulk metals. ${ }^{5}$ Remarkable progress has been reported over recent years in their synthesis or applications (for recent reviews, see for example ref. 6-8). In particu-

\footnotetext{
${ }^{a}$ Institut Lumière Matière, UMR CNRS 5306 and Université Claude Bernard Lyon 1, Université de Lyon, 69622 Villeurbanne cedex, France.

E-mail: rodolphe.antoine@univ-lyon1.fr

${ }^{b}$ Center of excellence for Science and Technology-Integration of Mediterranean region (STIM) at Interdisciplinary Center for Advanced Sciences and Technology (ICAST), University of Split, Meštrovićevošetalište 45, HR-2100 Split, Republic of Croatia ${ }^{c}$ Faculty of Science, University of Split, Teslina 12, HR-2100 Split, Republic of Croatia ${ }^{d}$ Department of Chemistry, Humboldt Universitatzu Berlin, Brook-Taylor-Strasse 2, 12489 Berlin, Germany

$\dagger$ Electronic supplementary information (ESI) available: CID spectrum of $\mathrm{Ag}_{29}$ (DHLA) $)_{12}$, spectroscopic characterization of the synthesized Ag clusters, lifetime measurements of silver cluster, schematic diagram showing the excited state relaxation dynamics, HRS intensity versus wavelength for $\mathrm{Ag}_{29}$ (DHLA) ${ }_{12}$, DFT optimized structures and TDDFT calculated one photon absorption spectra of $\mathrm{Ag}_{29}$ (DHLA $)_{12}$, Leading excitations for transition for prototype structures twophoton cross section for $\mathrm{Ag}_{29}$ (DHLA) ${ }_{12}$ at different wavelengths and optimal setting for the ESI-MS. See DOI: 10.1039/c5nr08122j
}

lar, the synthetic route using templates to stabilize low nuclearity silver clusters is one of the most promising. Different scaffolds used as stabilizers for silver nanoclusters have been proposed, including dendrimers, ${ }^{9}$ DNA oligomers, ${ }^{10}$ cryogenic noble gas matrixes, ${ }^{11}$ or inorganic glasses. ${ }^{12}$

Ligands containing thiol groups are good candidates to stabilize silver NCs due to the high energy of the sulfur-silver bond. Kitaev and co-workers ${ }^{13,14}$ have initiated this field using chiral thiols to build these silver clusters. More recently, Bigioni and co-workers, ${ }^{15}$ Heiz and coworkers ${ }^{16}$ and our group $^{17}$ have reported the synthesis of glutathione stabilized magic-number silver cluster compounds. Interestingly, Roy et al. ${ }^{18}$ have reported for these clusters with different size blue, green and red emission through a mere temperature or silver salt concentration change during synthesis. These highly fluorescent properties ${ }^{17-20}$ were however counterbalanced by polydispersity, uncharacterization or poor photo-stability. Another thiolated ligand, namely dihydrolipoic acid (DHLA), also leads to highly fluorescent silver clusters, emitting in the red part of the optical spectrum and having a long period stability. ${ }^{19,21}$ Nonetheless, the preparation of sized silver quantum clusters with different wavelength range fluorescence emission using the same synthesis conditions like solvent, silver salt precursor, stabilizing ligand or reducing agent remains a challenging issue.

While progresses aiming at producing silver clusters with attractive features including brightness, tunability and photostability have been made recently, nonlinear optics may be an 
alternative route to tunability. Indeed, the different selection rules of one-photon and two-photon excitations may provide contrasted radiative properties due to the different excited states involved. In their pioneering work, Dickson et al. have thus shown that two-photon excited fluorescence (TPEF) spectra of oligonucleotide-encapsulated silver clusters were blue-shifted with respect to the corresponding one-photon excited fluorescence (OPEF) ones, indicating that with TPEF, higher excited electronic states different than those reached by OPEF were involved. ${ }^{22}$ Also, Polavarapu et al. ${ }^{23}$ have successfully demonstrated that water soluble glutathione capped small gold clusters can be used for live cell imaging with both OPEF and TPEF. On our side, we have recently reported enhanced two-photon absorption cross sections and first hyperpolarizabilities for protected $\mathrm{Au}_{15}$ and $\mathrm{Au}_{25}$ clusters. ${ }^{24}$ Theoretical attempts to reproduce enhanced TPA cross-sections and first hyperpolarizabilities of thiolated-protected gold clusters were also recently reported..$^{25,26}$

In this work, we investigate the nonlinear optical (NLO) properties of DHLA capped 29 atom silver clusters upon twophoton excitation. We have thus determined the two-photon excited fluorescence spectra and the first hyperpolarizability for these clusters. We then show that the tuning of the fluorescence emission from the red to the blue side of the optical spectrum can be trigged by a one or a two-photon excitation.

\section{Results and discussion}

\section{Synthesis and characterization}

The silver clusters synthesis is based on the method already published by Adhikari et al. ${ }^{19}$ However, this synthesis led to highly sodated silver clusters ${ }^{21}$ preventing the easy assignment of their composition by mass spectrometry. Therefore, in order to obtain mass spectra without sodium adducts, $\mathrm{NaBH}_{4}$ and $\mathrm{NaOH}$ were replaced with tetramethylammonium borohydride $\left(\mathrm{TMABH}_{4}\right)$ and $\mathrm{NH}_{4} \mathrm{OH}$ respectively (see Materials and methods section). The solution of the as-prepared silver clusters has an orange color. The size of the prepared silver clusters was assigned using ESI-mass spectrometry, a particularly useful technique in the case of silver clusters due to their unequivocal isotopic pattern. An ESI mass spectrum of the asprepared DHLA protected silver clusters acquired under gentle ESI conditions is shown in Fig. 1 (see Table ST1 in ESI $\uparrow$ for the optimal parameters for gentle ionization conditions of ESI-MS). A charge state distribution was observed ranging from $[\mathrm{M}-2 \mathrm{H}]^{2-}$ to $[\mathrm{M}-5 \mathrm{H}]^{5-}$ along with peaks at lower masses corresponding to the neutral loss of $208 \mathrm{Da}$. The ESI spectra in the inset of Fig. 1 show the isotopic resolution for the 3- charge state. The measured mass distribution is compared with a simulated isotopic distribution of the $\left[\mathrm{Ag}_{29}(\mathrm{DHLA})_{12}-3 \mathrm{H}\right]^{3-}$ cluster. The agreement between the experimental and simulated distributions is found to be good. The observation reported here for the $\mathrm{Ag}_{29}$ clusters is different from the assignment previously reported for the $\mathrm{Ag}$ to $\mathrm{Ag}_{5}$ and $\left[\mathrm{Ag}_{25}(\mathrm{DHLA})_{14}-x \mathrm{H}+x \mathrm{Na}\right]$ clusters. ${ }^{19,21}$ Different solvent com-

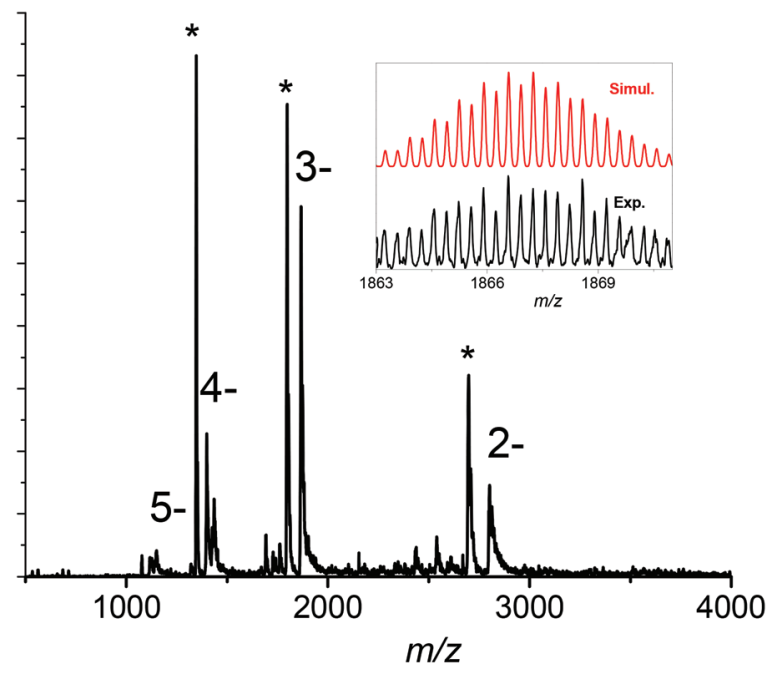

Fig. 1 ESI mass spectrum of the synthesized Ag clusters. Right inset: comparison of the experimental and simulated isotopic distributions for the 3 - charge state of the Ag clusters $\left(\left[\mathrm{Ag}_{29}(\mathrm{DHLA})_{12}-3 \mathrm{H}\right]^{3-}\right)$. The asterisk corresponds to peaks assigned to the loss of one DHLA molecule from the Ag clusters (see text for details).

position and different reducing agents may give different stoichiometry of Ag nanoclusters as those reported in ref. 21. Note that single DHLA ligand can be bound to more than one Ag atom and the core of several Ag atoms can be formed as discussed below. Interestingly, using bidentate ligands like 1,3benzenedithiol (BDT) in conjunction with triphenylphosphine (TPP), a tetravalent $\mathrm{Ag}_{29}(\mathrm{BDT})_{12}(\mathrm{TPP})_{4}$ nanocluster can be synthesised, readily assembling to form well-defined macroscopic crystals enabling its structure determination. ${ }^{27}$

The peaks relative intensity corresponding to the neutral loss of 208 Da was found to strongly depend on the ESI source parameters and ionic train voltages. This suggests that these ions arise from the $\left[\mathrm{Ag}_{29}(\mathrm{DHLA})_{12}-3 \mathrm{H}\right]^{3-}$ fragmentation. To validate this hypothesis, we performed collision-induced dissociations in the ion trap. Fig. S1† shows the CID spectrum of the $\left[\mathrm{Ag}_{29}(\mathrm{DHLA})_{12}-3 \mathrm{H}\right]^{3-}$ cluster. The observed $208 \mathrm{Da}$ intense loss is attributed to the loss of one DHLA ligand molecule $\left(\mathrm{C}_{8} \mathrm{H}_{16} \mathrm{O}_{2} \mathrm{~S}_{2}\right)$. Note also that CID pathways for liganded clusters involve the formation of $\mathrm{Ag}_{n}(\mathrm{DHLA})_{m}$ cluster ion products, see Fig. S1 in ESI. $\uparrow$ The stoichiometry observed here is different from the one reported for gluthatione-protected gold clusters and may be attributed to the fact the DHLA possesses two sulphur atoms leading to different staple motifs as discussed below.

\section{Linear optical properties}

The optical properties of the DHLA protected Ag clusters were characterized by absorption spectroscopy in the UV-visible-NIR range. The absorption spectrum, shown in Fig. 2, shows several sharp transition bands with maxima at 328, 428 and $499 \mathrm{~nm}$ combined with a shallow absorbance onset around $600 \mathrm{~nm}$. Photoluminescence excitation spectra of dilute silver nanocluster colloidal solutions at room temperature are also 


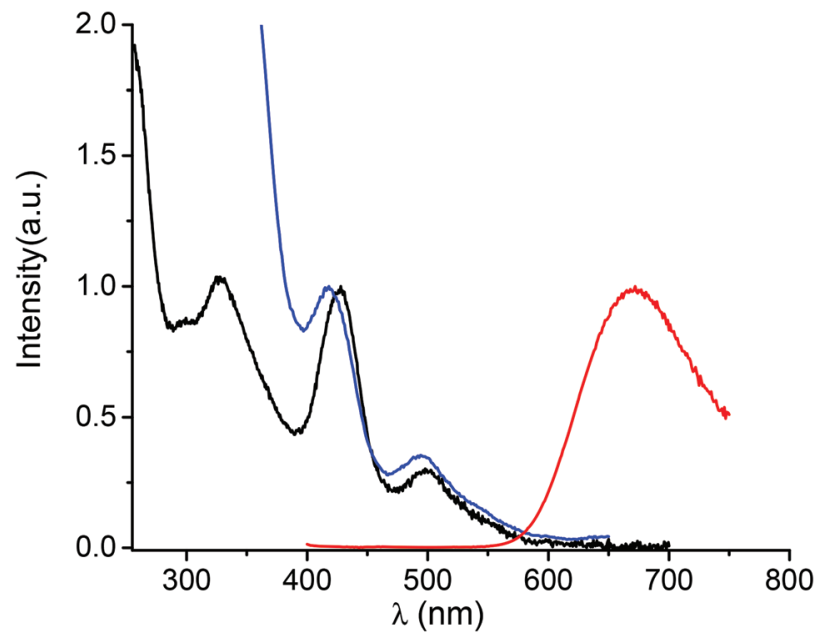

Fig. 2 Spectroscopic characterization of the synthesized Ag clusters dispersed in water. Absorption spectrum (black), fluorescence spectrum (red) with excitation at $385 \mathrm{~nm}$ and photoluminescence excitation spectrum (blue) with emission at $670 \mathrm{~nm}$.

presented in Fig. 2. The photoluminescence spectra show emission maxima at $\sim 670 \mathrm{~nm}$ upon excitation at $385 \mathrm{~nm}$. No significant change in the emission maximum was observed when changing the excitation wavelength (from $385 \mathrm{~nm}$ to $500 \mathrm{~nm}$ ), see Fig. S2 in ESI. $\dagger$ The luminescence quantum yield was measured to be $2 \pm 1 \%$ using Nile Blue as the reference dye (see the Materials and methods section for details). In order to have a better understanding of the excited-state dynamics, we performed picosecond time-resolved fluorescence decay measurements for silver clusters. Results are given in Fig. S3 in ESI. $\uparrow$ The decay curve can be fitted by a triple exponential curve, with a long decay time of $951 \mathrm{~ns}$. Light emission through ligand-to-metal or ligand-to-metalmetal charge transfer (LMCT/LMMCT) always shows a long fluorescence lifetime, partly because the LMCT/LMMCT can affect the excited state relaxation dynamics. Therefore, the observed emission is thought to arise from $\mathrm{Ag}(\mathrm{I})$ states on the nanocluster surface. These $\mathrm{Ag}(\mathrm{I})$ states may be present as $\mathrm{Ag}(\mathrm{I})$ thiolate complexes capping a $\operatorname{Ag}(0)$ core, a structural feature that has been found for thiolated gold nanoclusters. ${ }^{28}$ The linear optical properties reported here are similar to those presented in ref. 19 and 21.

\section{Nonlinear optical properties}

TPEF spectra with excitation at $790 \mathrm{~nm}$ and $950 \mathrm{~nm}$ were recorded for the $\mathrm{Ag}_{29}(\mathrm{DHLA})_{12}$ clusters, see Fig. 3. A broad band in the visible range, between 400 and $650 \mathrm{~nm}$, is observed upon excitation at $790 \mathrm{~nm}$ whereas for an excitation at $950 \mathrm{~nm}$, the TPEF spectrum is red shifted between 550 and $750 \mathrm{~nm}$. These TPEF spectra drastically contrast with their linear OPEF counterpart where emission in the red only, between 550 and $750 \mathrm{~nm}$ is observed, see Fig. 2. The latter OPEF spectrum closely resembles the TPEF one with excitation at $950 \mathrm{~nm}$. However, it is clearly observed that a tuning of the

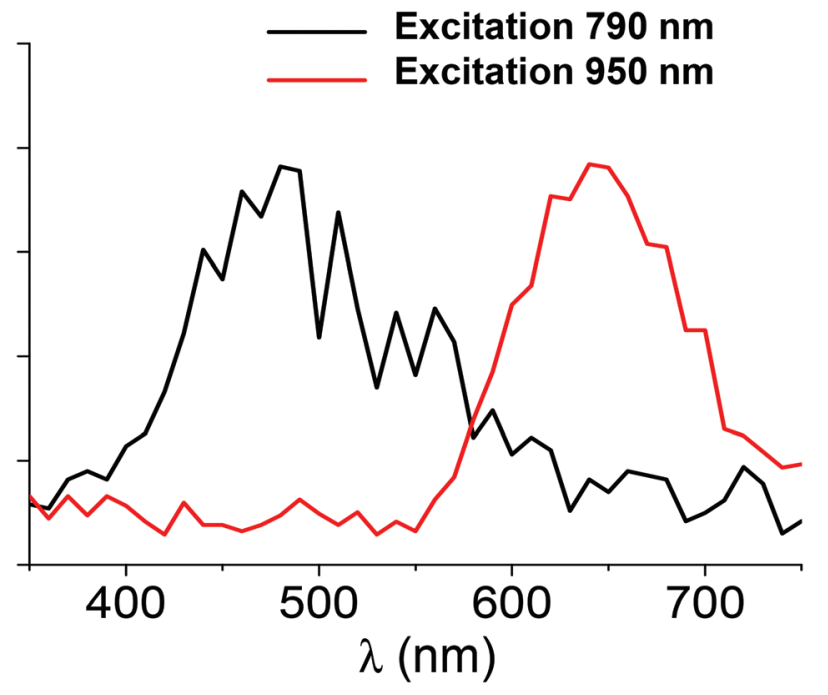

Fig. 3 Two-photon excited fluorescence spectra for Ag clusters as a function of wavelength for the two excitation wavelengths of $790 \mathrm{~nm}$ and $950 \mathrm{~nm}$.

emission from red to blue can be trigged by the appropriate choice of the excitation, either a one photon excitation or a two photon excitation with $790 \mathrm{~nm}$ light. Such a difference between the emission resulting from one or two photon absorption has already been reported by Dickson et al. ${ }^{22}$ for DNA-protected silver clusters and organic dyes. ${ }^{29}$ The relative transition strengths for OPEF and TPEF are indeed highly symmetry dependent because they do not obey the same transition rules. Interestingly, the TPEF spectrum upon excitation at $950 \mathrm{~nm}$ is strongly red-shifted and compares well with the one reported upon excitation at $790 \mathrm{~nm}$, see Fig. 3. Upon excitation at $950 \mathrm{~nm}$ for the TPEF spectrum and 426 or $498 \mathrm{~nm}$ for the OPEF one, both emission spectra appear similar, see Fig. S2 in ESI. $\uparrow$ The difference observed between the OPEF and TPEF spectra may therefore be accounted for the different symmetry rules required for the metal-to-metal excitations within the silver cluster core and the LMCT/LMMCT excitations as shown in the excitation pathway scheme given in Fig. S4 of the ESI. $\dagger$

We attempted to determine the two-photon absorption and emission cross-sections for these $\mathrm{Ag}_{29}$ (DHLA) ${ }_{12}$ nanoclusters, using the methods reported in our previous work. ${ }^{24}$ Experimentally, we found that the TPEF cross-section for a $790 \mathrm{~nm}$ excitation is $0.5 \mathrm{GM}$ and the TPA cross-section $12930 \mathrm{GM}$. These experimental cross-sections point to a quantum yield $\left(\mathrm{QY}=\sigma_{\mathrm{TPE}} / \sigma_{\mathrm{TPA}}\right)$ for $\mathrm{Ag}_{29}(\mathrm{DHLA})_{12}$ nanoclusters of about $4 \times$ $10^{-5}$, see Table ST2 in ESI $\dagger$ for the experimental cross-sections at different wavelengths. As already observed for gold nanoclusters, silver protected clusters are excellent two-photon absorbers but present a limited two-photon emission cross section due to a rather low quantum yield. Nevertheless, the two photon quantum yield is about two orders of magnitude higher for silver clusters than for the gold ones. First hyperpolarizabilities of these $\mathrm{Ag}_{29}$ (DHLA $)_{12}$ nanoclusters were also obtained using the hyper-Rayleigh scattering (HRS) technique. 
The HRS intensity was recorded for several concentrations of the $\mathrm{Ag}_{29}(\mathrm{DHLA})_{12}$ clusters dispersed in aqueous solutions, see Fig. S5 in ESI. $\uparrow$ The first hyperpolarizability $\beta$ determined for an excitation wavelength of $800 \mathrm{~nm}$ for the $\mathrm{Ag}_{29}$ (DHLA $)_{12}$ clusters is found to be $106 \times 10^{-30}$ esu, close to the value reported for $\mathrm{Au}_{25}$ clusters, i.e. $128 \times 10^{-30}$ esu. $^{24}$ Beside the exact number of atoms in the cluster metallic core, the ligands also participate to the rather large first hyperpolarizabilities, either directly with an intrinsic contribution or indirectly by imprinting symmetry changes on the core.

\section{Calculated structure and absorption spectra of protected silver nanocluster}

Structures of $\mathrm{Ag}_{29}(\mathrm{DHLA})_{12}$ are composed from DHLA ligands in which two sulfur atoms are bound to different number of $\mathrm{Ag}$ atoms, such that silver cores of different sizes can be formed. In the case that the central $\mathrm{Ag}$ atom is present, the symmetric structure (point group $\mathrm{T}$ ) is formed. However, in the case of the neutral species, deviation from point group $\mathrm{T}$ for $\mathrm{Ag}_{13}$ subunit occurs due to odd degenerate orbitals are not fully occupied due to odd number of electrons which do not correspond to superatom occupancy. For the structure with central Ag atom, two sulfur atoms of DHLA are bound to two and three $\mathrm{Ag}$ atoms respectively, forming $\mathrm{Ag}_{13}$ subunit related to centered icosahedral structure in which twelve $\mathrm{Ag}$ atoms are bound to twelve $\mathrm{S}$ atoms ( $c f$. right side of Fig. S6†).

In contrast, if the central atom is not present, other structural class arise with higher energy. For example, the core of six Ag atoms as capped trigonal bipyramid can be formed, in which S atoms from DHLA are not bound to Ag atoms of the core. In this case, the symmetry of structure is removed. Each $\mathrm{S}$ atom is bound to one or two $\mathrm{Ag}$ atoms not belonging to the core, as illustrated on the right side of Fig. S6. $\uparrow$ In this case the interface between the silver core and the ligand staples (S-Ag-S) contains also silver atoms forming an additional ligand shell. Similar situation occurs for structures containing two or five silver atoms as illustrated on Fig. S6. $\dagger$

Search for the global minimum is very demanding and has not been yet ensured. Moreover, calculations of optical properties for systems with odd number of electrons, such as $\mathrm{Ag}_{29}(\mathrm{DHLA})_{12}$ and for the structures with almost degenerate HOMO orbitals that are not fully occupied are methodologically difficult. Therefore, we have calculated absorption properties for two classes of structures: (A) containing central Ag atom and almost symmetrical $\mathrm{Ag}_{13}$ subunit and (B) structures with silver core of different sizes containing interface of silver atoms as described above. Comparison of TDDFT calculated absorption features for the lowest energy structure belonging to class (A) with the measured experimental absorption spectra, as shown in Fig. 4, illustrates good qualitative agreement, exhibiting characteristic absorption features between 300 and $500 \mathrm{~nm}$ containing pronounced maximum at $420 \mathrm{~nm}$ which arises due to excitations involving $\mathrm{Ag}_{13}$ subunit as well as ligands. Since two photons excitations can occur through resonance with the low lying one-photon excited states, resonance conditions for large TPA cross-sections can be easily ful-

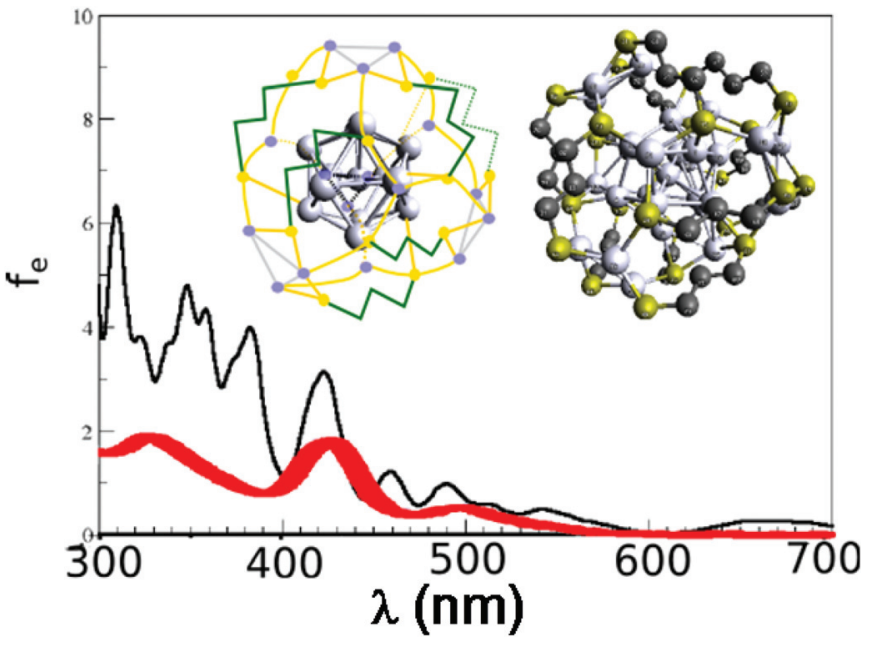

Fig. 4 Comparison of experimental (red line) and theoretical absorption spectra (black line) obtained by PAW TDDFT for optimised structure with $\mathrm{Ag}_{13}$ subunit (see computational for details). Right inset: DFT optimized structure of $\mathrm{Ag}_{29}(\mathrm{DHLA})_{12}$. The DHLA is represented by $\mathrm{S}-\left(\mathrm{CH}_{2}\right)_{3}-\mathrm{S}$ and carboxylic tail is replaced by one $\mathrm{H}$ atom. Silver atoms are labelled by grey balls bound to $\mathrm{S}$ atoms (yellow balls). Middle insert: illustrative scheme of the optimized structure shown in the right inset.

filled, as it has been experimentally found. Comparison of absorption properties for the lowest energy structure containing central $\mathrm{Ag}$ atom to those belonging to structural class (B) are shown on the left side of Fig. S6. $\dagger$ The excitations responsible for the transition close to $420 \mathrm{~nm}$ for both prototype of structural classes, involving silver subunit or core and ligands are shown in Fig S7. $\dagger$ The agreement with experimental findings is slightly better for structural class (A).

All together $\mathrm{Ag}_{29}(\mathrm{DHLA})_{12}$ owns novel properties due to its electronic structure with odd number of electrons and relatively low HOMO-LUMO gap giving rise to low energy onephoton excited states which are also reachable by two-photons. This indicates that the search for liganded silver species, in which various sizes of metal core can be formed giving rise to different structural classes might lead towards design of systems with unique linear and non-linear properties useful in context of different applications.

\section{Conclusions}

Atomically size-controlled synthesis of monodispersed silver nanoclusters offers the possibility to prepare hybrid metalorganic nanoclusters with a well-defined stoichiometry. Their linear and nonlinear optical properties can then be finely compared. It appears then that the different selection rules involved for one- and two-photon excitations provide interesting radiative features in their emission spectra, notably because different excited states are accessible by one- and twophoton excitation depending on the wavelength of irradiation. We have found in particular that for the $\operatorname{Ag}_{29}(\mathrm{DHLA})_{12}$ 
nanoclusters, the TPEF spectrum is strongly blue-shifted with $790 \mathrm{~nm}$ excitation as compared to the OPEF spectrum and the TPEF one with excitation at $950 \mathrm{~nm}$. It displays a broad emission band between 400 and $700 \mathrm{~nm}$ instead of the usual emission band between 550 and $750 \mathrm{~nm}$. Structural and electronic properties of $\mathrm{Ag}_{29}(\mathrm{DHLA})_{12}$ nanocluster opens the route for recognizing the important role of ligands and their interface with metallic subunits for inducing unique linear and non-linear optical properties in these systems. These newly prepared bright fluorescence silver nanoclusters hold an interesting promise in the future for contrasted biolabeling, biosensing or other applications where OPEF and TPEF wavelength tuning is requested.

\section{Materials and methods}

\section{Synthesis}

Silver nitrate $\left(\mathrm{AgNO}_{3}\right),( \pm)$ - $\alpha$-lipoic acid and tetramethylammonium borohydride $\left(\left(\mathrm{CH}_{3}\right)_{4} \mathrm{NBH}_{4}\right)$ were purchased from Sigma Aldrich. In a $100 \mathrm{ml}$ flask, $100 \mathrm{mg}$ of $( \pm)$ - $\alpha$-lipoic acid is dissolved in $5 \mathrm{ml}$ of methanol and $10 \mathrm{ml}$ of water are added followed by 2 equivalents $(85 \mathrm{mg}$ ) of tetramethylammonium borohydride (under agitation $30 \mathrm{~min}$ ) to reduce lipoic acid. Then, excess of reducer is eliminated by sonication (1 hour). $41 \mathrm{mg}$ of silver nitrate (dissolved in $15 \mathrm{ml}$ of water) is quickly added, followed by $1 \mathrm{ml}$ of $10 \% \mathrm{NH}_{4} \mathrm{OH}$ solution. The solution is heated at $50{ }^{\circ} \mathrm{C}$ and 2 equivalents of tetramethylammonium borohydride are added ( $85 \mathrm{mg}$ ) and stirred. After one hour the color becomes bright orange and one equivalent of tetramethylammonium borohydride $(42.5 \mathrm{mg})$ is added. After one hour, the solution is concentrated to $\approx 1 \mathrm{ml}$ by using a rotary evaporator and transferred into a centrifuge tube. $5 \mathrm{ml}$ of ethanol and $20 \mathrm{ml}$ of diethyl ether are added, followed by $50 \mu \mathrm{l}$ of glacial acetic acid. The precipitate was centrifuged 5 minutes at $9000 \mathrm{rpm}$ and the supernatant subsequently removed. The precipitate is washed first with ethanol/diethyl ether and then with diethyl ether alone. The resulting brown powder is dried.

\section{Mass spectrometry}

Solutions were diluted without gel separation at a concentration of $\sim 1 \mathrm{mg} \mathrm{ml}^{-1}$ in water, electrosprayed at a flow rate of

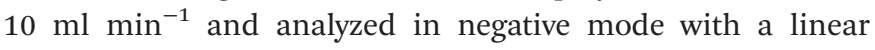
quadrupole ion trap mass spectrometer (LTQ, Thermo Fisher Scientific, San Jose, CA) with a spray voltage of $3 \mathrm{kV}$ and a capillary temperature of $150{ }^{\circ} \mathrm{C}$. Other instrument settings were adjusted for each species to optimize the distribution of charge states observed in the mass spectrum (see Table ST1 in ESI $\dagger$ for the optimal parameters for gentle ionization conditions of ESI-MS). Isotope-resolved mass spectra were recorded using the zoom scan mode of the instrument (mass resolution $\sim 10000$, mass accuracy $<0.2 \mathrm{Th}$ ). Collision-induced dissociation (CID) was performed using helium gas at a normalized collision energy of $20 \%$ for $30 \mathrm{~ms}$.

\section{UV/visible and fluorescence measurements}

UV-vis spectra in solution were recorded using an AvaSpec2048 fiber optic spectrometer and an AvaLight-DH-S deuterium-halogen light source. Fluorescence excitation and emission spectra and lifetime measurements were obtained using a Fluoromax-4 Horiba fluorescence spectrophotometer in the time resolved mode. Luminescence decay curves were collected with the time-correlated single-photon counting (TCSPC) extension of Fluoromax-4 using a nanoLED source at $370 \mathrm{~nm}$.

\section{Quantum yield measurement}

Nile Blue dissolved in methanol (quantum yield of 0.27$)^{30}$ used as a standard and $500 \mathrm{~nm}$ was chosen as the excitation wavelength. The emission intensities of Nile Blue and the silver nanoclusters were integrated from $500 \mathrm{~nm}$ up to $850 \mathrm{~nm}$. Both the excitation and the emission slit apertures were $5 \mathrm{~mm}$.

\section{HRS and TPE measurements}

The light source for the HRS, TPA and TPE experiments was a mode-locked femtosecond Ti:sapphire laser delivering at the fundamental wavelength of $780 \mathrm{~nm}$ and $950 \mathrm{~nm}$ pulses with a duration of about 140 femtoseconds at a repetition rate of $76 \mathrm{MHz}$. After passing through a low-pass filter to remove any unwanted harmonic light generated prior to the cell, the fundamental beam of about $300 \mathrm{~mW}$ was focused by a microscope objective into a $0.5 \mathrm{~cm}$ spectrophotometric cell containing the aqueous solutions. The HRS and TPEF light were collected at an angle of $90^{\circ}$ from the incident direction by a $2.5 \mathrm{~cm}$ focal length lens. The HRS light was separated from the excitation light by a high-pass filter and a monochromator position at the second harmonic wavelength. The HRS light was then detected with a photomultiplier tube working in the single photon counting regime. For the TPE signal, the wavelength of the spectrometer (iHR320 spectrometer) was varied between $350 \mathrm{~nm}$ and $750 \mathrm{~nm}$.

\section{TPA experimental arrangement}

The light source for the present TPA experiments was the same as the above. The beam was gently focused by a $5 \mathrm{~cm}$ focal lens and sent in transmission into a $0.5 \mathrm{~cm}$ path length spectrophotometric cuvette. The transmitted light was detected with a large aperture photodiode. The incident power was controlled with a half-wave plate and a polarizing cube. The sample absorption, the concentration of which was set to $500 \mu \mathrm{M}$, was then determined as a function of the incident power. Fluorescein was used as a reference to determine the beam waist at focus in particular. The calibration of the photodiode signal was obtained with a neat water cuvette through a variation of the incident power. As expected with the neat water cuvette, the plot of the transmitted light versus the incident light exhibits a linear behavior whereas in the presence of the silver nanoclusters, a decrease of the transmitted intensity is observed as the absorption increases due to the nonlinear contribution. 


\section{Computational}

Different level of DFT has been used to determine structural properties of $\mathrm{Ag}_{29}(\mathrm{DHLA})_{12}$. For this purpose, a DFT approach with Perdew-Burke-Ernzerhof functional (PBE), ${ }^{31}$ and resolution of identity (RI-approximation) ${ }^{32}$ has been employed. Silver atoms were treated with Stuttgart relativistic core (RECP) potential with corresponding basis set. ${ }^{33}$ In extensive search for the lowest energy structures within Turbomole codepackage, ${ }^{34}$ also crystal structure $\mathrm{Ag}_{29}(\mathrm{BDT})_{12}(\mathrm{TPP})_{4}{ }^{27}$ has served as one of the guidance routes because the search for global minimum is very difficult for this size of the system. Therefore, structural properties have been also investigated with projector augmented waves (PAW) as implemented in real-space code-package GPAW $^{35,36}$ because it is less computationally demanding. Since the both DFT methods give rise to almost identical structures, the optical absorption spectra were also calculated by using linear response TDDFT as implemented in GPAW. ${ }^{37}$ The PAW setup was defined for atomic valence of $\mathrm{Ag}\left(4 \mathrm{~d}^{10} 5 \mathrm{~s}^{1}\right), \mathrm{S}\left(3 \mathrm{~s}^{2} 3 \mathrm{p}^{2}\right), \mathrm{C}\left(2 \mathrm{~s}^{2} 2 \mathrm{p}^{2}\right)$ and $\mathrm{H}\left(1 \mathrm{~s}^{1}\right)$ with scalar relativistic effects included for $\mathrm{Ag}$. The PBE exchange correlation functional was employed with real space grid spacing of $0.2 \mathrm{~A}$ and $0.05 \mathrm{eV} \AA^{-1}$ convergence criteria for residual forces acting on atoms for calculation of ground state energy and structural properties. For the absorption properties, the spacing in real space grid was $0.2 \AA$ and Gaussian broadening of $0.04 \mathrm{eV}$ has been used.

\section{References}

1 V. Bonacic-Koutecky, A. Kulesza, L. Gell, R. Mitric, R. Antoine, F. Bertorelle, R. Hamouda, D. Rayane, M. Broyer, T. Tabarin and P. Dugourd, Phys. Chem. Chem. Phys., 2012, 14, 9282-9290.

2 S. Choi, R. M. Dickson and J. Yu, Chem. Soc. Rev., 2012, 41, 1867-1891.

3 W. Chen, in Silver Nanoparticles: Synthesis, Uses and Health Concerns, ed. I. Armentano and J. M. Kenny, Nova Science Publishers, Inc., 2013.

4 S. M. Copp, D. E. Schultz, S. Swasey and E. G. Gwinn, ACS Nano, 2015, 9, 2303-2310.

5 R. C. Jin, Nanoscale, 2010, 2, 343-362.

6 I. Diez and R. H. A. Ras, Nanoscale, 2011, 3, 1963-1970.

7 T. Udayabhaskararao and T. Pradeep, J. Phys. Chem. Lett., 2013, 4, 1553-1564.

8 K. Zheng, X. Yuan, N. Goswami, Q. Zhang and J. Xie, RSC Adv., 2014, 4, 60581-60596.

9 J. Zheng and R. M. Dickson, J. Am. Chem. Soc., 2002, 124, 13982-13983.

10 J. T. Petty, J. Zheng, N. V. Hud and R. M. Dickson, J. Am. Chem. Soc., 2004, 126, 5207-5212.

11 W. Harbich, S. Fedrigo and J. Buttet, Chem. Phys. Lett., 1992, 195, 613-617.

12 R. Espiau de Lamaestre, H. Béa, H. Bernas, J. Belloni and J. L. Marignier, Phys. Rev. B: Condens. Matter, 2007, 76, 205431.
13 N. Cathcart and V. Kitaev, J. Phys. Chem. C, 2010, 114, 16010-16017.

14 N. Cathcart, P. Mistry, C. Makra, B. Pietrobon, N. Coombs, M. Jelokhani-Niaraki and V. Kitaev, Langmuir, 2009, 25, 5840-5846.

15 S. Kumar, M. D. Bolan and T. P. Bigioni, J. Am. Chem. Soc., 2010, 132, 13141-13143.

16 M. Farrag, M. Tschurl and U. Heiz, Chem. Mater., 2013, 25, $862-870$.

17 F. Bertorelle, R. Hamouda, D. Rayane, M. Broyer, R. Antoine, P. Dugourd, L. Gell, A. Kulesza, R. Mitric and V. Bonacic-Koutecky, Nanoscale, 2013, 5, 5637-5643.

18 S. Roy, A. Baral and A. Banerjee, ACS Appl. Mater. Interfaces, 2014, 6, 4050-4056.

19 B. Adhikari and A. Banerjee, Chem. Mater., 2010, 22, 43644371.

20 X. Le Guével, C. Spies, N. Daum, G. Jung and M. Schneider, Nano Res., 2012, 5, 379.

21 P. T. K. Chin, M. van der Linden, E. J. van Harten, A. Barendregt, M. T. M. Rood, A. J. Koster, F. W. B. van Leeuwen, C. de Mello Donega, A. J. R. Heck and A. Meijerink, Nanotechnology, 2013, 24, 075703.

22 S. A. Patel, C. I. Richards, J.-C. Hsiang and R. M. Dickson, J. Am. Chem. Soc., 2008, 130, 11602-11603.

23 L. Polavarapu, M. Manna and Q.-H. Xu, Nanoscale, 2011, 3, 429-434.

24 I. Russier-Antoine, F. Bertorelle, M. Vojkovic, D. Rayane, E. Salmon, C. Jonin, P. Dugourd, R. Antoine and P.-F. Brevet, Nanoscale, 2014, 6, 13572-13578.

25 P. N. Day, K. A. Nguyen and R. Pachter, J. Chem. Theory Comput., 2010, 6, 2809-2821.

26 S. Knoppe, H. Häkkinen and T. Verbiest, J. Phys. Chem. C, 2015, 119(49), 27676-27682.

27 L. G. AbdulHalim, M. S. Bootharaju, Q. Tang, S. Del Gobbo, R. G. AbdulHalim, M. Eddaoudi, D.-E. Jiang and O. M. Bakr, J. Am. Chem. Soc., 2015, 137, 11970-11975.

28 Z. K. Wu and R. C. Jin, Nano Lett., 2010, 10, 2568-2573.

29 C. Xu and W. W. Webb, J. Opt. Soc. Am. B, 1996, 13, 481491.

30 R. Sens and K. H. Drexhage, J. Lumin., 1981, 24, 709-712.

31 J. P. Perdew, K. Burke and M. Ernzerhof, Phys. Rev. Lett., 1996, 77, 3865-3868.

32 K. Eichkorn, O. Treutler, H. Öhm, M. Häser and R. Ahlrichs, Chem. Phys. Lett., 1995, 242, 652-660.

33 D. Andrae, U. Haeussermann, M. Dolg, H. Stoll and H. Preuss, Theor. Chim. Acta, 1990, 77, 123.

34 TURBOMOLE V6.2 2010, a development of University of Karlsruhe and Forschungszentrum Karlsruhe $\mathrm{GmbH}$ and T. G. 1989-2007, since 2007; available from http://www. turbomole.com.

35 J. Enkovaara, C. Rostgaard, J. J. Mortensen, J. Chen, M. Dułak, L. Ferrighi, J. Gavnholt, C. Glinsvad, V. Haikola, H. A. Hansen, H. H. Kristoffersen, M. Kuisma, A. H. Larsen, L. Lehtovaara, M. Ljungberg, O. LopezAcevedo, P. G. Moses, J. Ojanen, T. Olsen, V. Petzold, N. A. Romero, J. Stausholm-Møller, M. Strange, 
G. A. Tritsaris, M. Vanin, M. Walter, B. Hammer, H. Häkkinen, G. K. H. Madsen, R. M. Nieminen, J. K. Nørskov, M. Puska, T. T. Rantala, J. Schiøtz, K. S. Thygesen and K. W. Jacobsen, J. Phys.: Condens. Matter, 2010, 22, 253202.
36 J. J. Mortensen, L. B. Hansen and K. W. Jacobsen, Phys. Rev. B: Condens. Matter, 2005, 71, 035109.

37 M. Walter, H. Häkkinen, L. Lehtovaara, M. Puska, J. Enkovaara, C. Rostgaard and J. J. Mortensen, J. Chem. Phys., 2008, 128, 244101. 